\title{
EDITORIAL
}

\section{Challenges for Indoor Air Quality Management}

\author{
Associate Professor dr. Violeta Kaunelienè
}

Department of Environmental Technology, Faculty of Chemical Technology, Kaunas University of Technology

We live indoors. In the developed countries, people spent $80-90 \%$ of time in buildings. Therefore, among the environmental factors, indoor air quality (IAQ) is a significant, if not the most significant, factor affecting human health. Scientific evidence has indicated that indoor air can be more seriously polluted than the outdoor air in even the largest and most industrialized cities. Indoor air pollution causes long- and short-term health problems and, depending on the severity, can even make staying indoors very uncomfortable. Management of IAQ is a complicated task due to complexity of pollution sources and a limited number of measures applicable such as reducing contaminants at the source, improving ventilation, and, when relevant, purifying the indoor air.

A good example to reduce pollutant emission at a source is a concept of the lowest concentration of interest $(\mathrm{LCl})$ for building materials which are one of the most significant sources of volatile organic compounds (VOCs). "EU-LCl are based on reported scientific data and expert judgment and represent concentration levels that are considered likely not to cause adverse effects over the longer term by use of the model room as a reference" (EC, 2020). However, the EU-LCl concept applies only to VOCs and does not cover semi VOCs despite of their abundancy in building products as well as their potential for endocrine disruption or carcinogenic properties. There is no similar emission prevention system for consumer products which are another important source of VOCs and SVOCs. Cooking, combustion based sources are significant contributors of both the particulate matter (PM) and VOCs. Tobacco smoke alone can contribute over 8000 different components (Rodgman and Perfetti, 2008).

The majority of buildings are limited to natural ventilation via windows or passive ventilation channels. The air exchange in such buildings is 2-3 times lower the recommended comfort value and is not capable to sufficiently dilute indoor air pollution. Only in buildings equipped with modern heating, ventilation and air conditioning (HVAC) systems, sufficient air exchange can be ensured without the loss of thermal energy. However, it is important to bear in mind that ventilation is a transporter of pollution with PM, nitrogen oxides, ozone, persistent organic pollutants like polyaromatic hydrocarbons or dioxins and other pollutants from outdoors unless an HVAC system is equipped with a proper air treatment unit. The air cleaning in the majority of HVAC systems is limited to filtration, often capable to remove only the coarse PM. Fine particles and gaseous pollutants pass through such systems.

Up to date no single physical-chemical technology is capable of coping with all indoor air pollutants in a cost effective manner. This problem requires the use of sequential technology configurations at the expenses of superior capital and operating costs (Gonzalez-Martín et al, 2021). Therefore, there remains a great need and prospect for technological improvement. Public awareness raising, however, can lead to substantial reduction of indoor air pollution from products with no or limited functionality such as tobacco smoke, excessive use of fragranced products, candles, incense, etc.

EC (European Commission) (2020). EU-LCl values. Available at: https://ec.europa.eu/growth/sectors/construction/eu-lcisubgroup/eu-lci-values_lt

Gonzalez-Martín J., Kraakman N. J. R., Perez C., Lebrero R. Munoz R. (2021). A state of the-art review on indoor air pollution and strategies for indoor air pollution control. Chemosphere, 262128376.

Rodgman, A., Perfetti T. A. (2008). The Chemical Components of Tobacco and Tobacco Smoke. CRC Press, 1840 p.p. 